\title{
Keratinocyte growth factor and the expression of wound-healing-related genes in primary human keratinocytes from burn patients ${ }^{1}$
}

\author{
Verônica Chomiski ${ }^{\mathrm{I}}$, Alfredo Gragnani ${ }^{\mathrm{II}}$, Jéssica Bonucci' ${ }^{\mathrm{II}}$, Silvana Aparecida Alves Correa ${ }^{\mathrm{IV}}$, Samuel Marcos Ribeiro de \\ Noronhav $^{v}$, Lydia Masako Ferreira ${ }^{\text {VI }}$
}

DOI: http://dx.doi.org/10.1590/S0102-865020160080000002

IFellow MSc degree, Division of Plastic Surgery, Department of Surgery, Universidade Federal de São Paulo (UNIFESP0, Sao Paulo-SP, Brazil. Acquisition and interpretation of data, technical procedures, manuscript writing.

IIPhD, Associate Professor, Division of Plastic Surgery, Department of Surgery, UNIFESP, Sao Paulo-SP, Brazil. Conception, design, intellectual and scientific content of the study; analysis and interpretation of data; manuscript writing; critical revision.

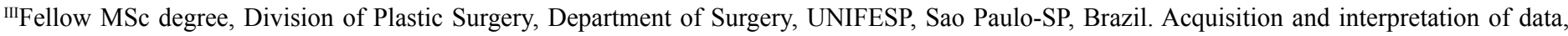
technical procedures.

${ }^{\mathrm{IV}} \mathrm{PhD}$, Postdoctoral degree, Division of Plastic Surgery, Department of Surgery, UNIFESP, Sao Paulo-SP, Brazil. Acquisition and interpretation of data, technical procedures, acquisition of data, manuscript writing.

${ }^{\mathrm{v}} \mathrm{PhD}$, Postdoctoral degree, Division of Plastic Surgery, Department of Surgery, UNIFESP, Sao Paulo-SP, Brazil. Acquisition and interpretation of data, manuscript writing.

${ }^{\mathrm{V}}$ Head, Full Professor, Division of Plastic Surgery, UNIFESP, Researcher 1A-CNPq, Director Medicine III-CAPES, Sao Paulo-SP, Brazil. Intellectual and scientific content of the study.

\section{ABSTRACT}

PURPOSE: To evaluate the effect of keratinocyte growth factor (KGF) treatment on the expression of wound-healing-related genes in cultured keratinocytes from burn patients.

METHODS: Keratinocytes were cultured and divided into 4 groups ( $\mathrm{n}=4$ in each group): TKB (KGF-treated keratinocytes from burn patients), UKB (untreated keratinocytes from burn patients), TKC (KGF-treated keratinocytes from controls), and UKC (untreated keratinocytes from controls). Gene expression analysis using quantitative polymerase chain reaction (qPCR) array was performed to compare (1) TKC versus UKC, (2) UKB versus UKC, (3) TKB versus UKC, (4) TKB versus UKB, (5) TKB versus TKC, and (6) UKB versus TKC.

RESULTS: Comparison 1 showed one down-regulated and one up-regulated gene; comparisons 2 and 3 resulted in the same five downregulated genes; comparison 4 had no significant difference in relative gene expression; comparison 5 showed 26 down-regulated and 7 up-regulated genes; and comparison 6 showed 25 down-regulated and 11 up-regulated genes.

CONCLUSION: There was no differential expression of wound-healing-related genes in cultured primary keratinocytes from burn patients treated with keratinocyte growth factor.

Key words: Burns. Gene Expression. Wound Healing. Keratinocytes. 


\section{Introduction}

Over 6 million people worldwide suffer a burn injury per year ${ }^{1}$. It is estimated that 1 million burn injuries occur annually only in the United States, accounting for 5,000 deaths in the same period. Burns are the fourth leading cause of death from unintentional injuries ${ }^{2}$. In Brazil, no official statistics provide estimates of burn incidence or mortality. However, burn injuries are not rare and can be costly to the public health system ${ }^{3}$.

Severe burn injury is a complex trauma associated with high morbidity and mortality, and immediate and long-term consequences, requiring intensive care in the acute treatment for restoration of hemodynamics, pain relief, preventing and treating sepsis, and skin replacement ${ }^{3,4}$. Burn patients also need special care due to psychosocial sequelae ${ }^{4}$.

Burn injuries affect not only the skin, but also other organs by inducing local and systemic responses. These injuries are associated with decreased tissue perfusion, prolonged hypotension, edema, infection, systemic hypermetabolism with extensive protein catabolism, and, eventually, irreversible tissue ${ }^{5}$. The high incidence of infection in burn patients is a major concern in clinical setting, because it is directly related to morbidity and mortality ${ }^{6,7}$

The inflammatory response is the first phase of tissue repair following an injury ${ }^{8}$. A thermal injury increases the levels of cytokines, which are the primary mediators in the inflammatory response involved in autocrine, paracrine, and endocrine pathways of cellular activation ${ }^{3,9}$. Antimicrobial peptides induced by the innate immune response from epithelial cells help protect the body through the activation of inflammatory cells or inactivation of pathogens, improving resistance to microbial infection ${ }^{8}$. However, prolonged inflammation hinders wound healing, because it results in excessively high levels of cytokines at the wound site, leading to collagen degradation, cellular apoptosis, vascular damage, and production of free radicals ${ }^{10}$. In the first 24 to 48 hours after an injury, a progressive deepening of wound takes place ${ }^{11}$. In the following days, extensive changes in gene expression of woundhealing-related cells may result in impaired re-epithelialization ${ }^{12,13}$. Methods for improving wound healing and tissue repair, and therapeutic interventions that attenuate the acute inflammatory response and cytokine expression after a burn injury may improve quality of life and reduce mortality and health care costs ${ }^{11,14,15}$.

A better understanding of the growth factors therapeutic value is essential to improve burn care ${ }^{16}$. Growth factors act on tissue repair by modulating the proliferation and migration of cells, and enhancing their metabolic activity ${ }^{16,17}$. Experimental and clinical studies support the therapeutic use of growth factors in wound healing.

Keratinocyte growth factor (KGF), also known as fibroblast growth factor 7 (FGF-7), has a unique role as a mediator of epithelial-mesenchymal interactions. The mechanism of KGF action was described by auf demKeller et al. ${ }^{18} \mathrm{KGF}$ is released by mesenchymal cells and acts only on epithelial cells. KGF is produced by fibroblasts and activates the IIIb isoform of the FGFreceptor 2 (FGFR2-IIIb) on keratinocytes. Receptor activation leads to migration, proliferation and survival of keratinocytes ${ }^{18}$. In vitro and in vivo studies have shown the KGF activity on epithelialization ${ }^{19-23}$. However, Wearing and Sherratt ${ }^{15}$ found that KGF added to the epidermis decreases re-epithelialization, although increases the signaling range of KGF.

According to modern biology, injuries cause alterations in the inflammatory response and may lead to changes in the gene expression of cells involved in this process. Quantitative analysis and comparisons of gene expression profiles in burn injuries provide good estimates of activation time, patterns of gene expression, and inflammatory responses ${ }^{12}$.

Considering that burn injuries trigger an intense inflammatory response due to alterations in gene expression, it is essential to understand the involved regulatory mechanisms and their role in tissue repair. Thus, the purpose of this study was to evaluate the effect of KGF treatment on the expression of woundhealing-related genes in cultured primary keratinocytes from burn patients to obtain an overview of the re-epithelialization process in this population.

\section{Methods}

The study was approved by the Institutional Research Ethics Committee (approval number 197.342) and carried out in accordance with The Code of Ethics of the World Medical Association (Declaration of Helsinki) for experiments involving humans. Patients were recruited at the Burn Treatment Unit and Plastic Surgery Unit of the Universidade Federal de São Paulo. Written informed consent was obtained from all patients prior to their inclusion in the study; patient privacy rights were observed.

Four hospitalized adult patients, on the third day postburn, of both genders with full-thickness or third-degree burn injuries affecting $25 \%$ to $50 \%$ of the total body surface area (TBSA) or referred to partial-thickness skin graft in $10 \%$ of the TBSA were included in this study. Skin biopsy specimens of burn patients were taken from upper limb or from chest. In addition, four healthy adult patients, who were candidates for aesthetic surgery 
(i.e., mammaplasty or abdominoplasty), were selected as controls. Eligibility criteria for controls included patients of both genders, non-smokers, and without history of diseases or medication use.

Skin biopsy specimens were obtained from all 8 participants and primary keratinocytes were cultured and divided into 4 groups: TKB $(\mathrm{n}=4, \mathrm{KGF}$-treated keratinocytes from burn patients); UKB ( $\mathrm{n}=4$, untreated keratinocytes from burn patients); TKC ( $\mathrm{n}=4$, KGF-treated keratinocytes from controls); and UKC $(\mathrm{n}=4$, untreated keratinocytes from controls).

Gene expression analysis using quantitative polymerase chain reaction (qPCR) array was performed for group comparisons (1) TKC versus UKC; (2) UKB versus UKC; (3) TKB versus UKC; (4) TKB versus UKB; (5) TKB versus TKC; and (6) UKB versus TKC.

\section{Surgical procedure}

Skin biopsy specimens were obtained from controls and burn patients by the plastic surgeon during standard aesthetic surgical procedures or during the surgical treatment of burns, respectively. Debridement of partial-thickness or fullthickness burns was performed at three days postburn. Normal skin surrounding the wound was removed as part of the surgical procedure.

Skin biopsy specimens were immediately immersed in a 50-mL Falcon tube containing $30 \mathrm{~mL}$ of Dulbecco's modified Eagle medium (DMEM) with high glucose (4.5 g/L), L-glutamine $(584 \mathrm{mg} / \mathrm{L})$, and sodium pyruvate $(110 \mathrm{mg} / \mathrm{L})$ (Gibco, Grand Island, USA), supplemented with penicillin-streptomycin (100 IU/ $\mathrm{mL}-100 \mu \mathrm{g} / \mathrm{mL}$ ) (Gibco). Samples were then immediately stored at $4^{\circ} \mathrm{C}$ and manipulated within 6 hours.

\section{Keratinocyte growth medium}

The keratinocyte growth medium containing $750 \mathrm{~mL}$ of DMEM and $250 \mathrm{~mL}$ of Ham's F-12 medium (Gibco) was supplemented with 10\% Fetal Bovine Serum (FBS) (Hyclone, Logan, USA) and $24 \mathrm{mg}$ of adenine (6-aminopurine hydrochloride; Sigma, St. Louis, USA) diluted with 20 mL of DMEM/Ham's F-12 solution to a concentration of $1.8 \times 10^{-4} \mathrm{~mol} / \mathrm{L}$. The growth medium was also supplemented with $1 \mathrm{~mL}$ of cholera toxin (Vibrio cholera, Type Inaba $569 \mathrm{~B}$ ) at $10^{-10} \mathrm{~mol} / \mathrm{L}$ (Gibco), $2 \mathrm{~mL}$ of penicillinstreptomycin $(100 \mathrm{IU} / \mathrm{mL}-100 \mu \mathrm{g} / \mathrm{mL}), 2 \mathrm{~mL}$ of hydrocortisone $(0.4 \mu \mathrm{g} / \mathrm{mL})$ (Sigma), $1 \mathrm{~mL}$ of transferrin/triiodo-L-thyronine $\left(2 \times 10^{-9} \mathrm{~mol} / \mathrm{L}\right)$ (Sigma), and $1.3 \mathrm{~mL}$ of porcine insulin $(5 \mathrm{mg} /$ $\mathrm{mL}$ ) (Sigma), totaling $5 \mu \mathrm{g} / \mathrm{mL}$ for the final concentration of the keratinocyte growth medium ${ }^{24,25}$.

\section{Isolation and culture of human primary epidermal keratinocytes}

Culture of human primary epidermal keratinocytes was performed following the protocol described by Green et al.24,25.

For separation of the epidermal sheet, the skin specimens were cut into small pieces of $0.5 \mathrm{~cm} \times 0.5 \mathrm{~cm}$ and placed into a $50-\mathrm{mL}$ Falcon tube containing $20 \mathrm{~mL}$ of dispase $(0.6-2.4 \mathrm{U} / \mathrm{mL})$ (Gibco) dissolved in DMEM26 for 18 hours. The specimens were then washed once with Versene (Gibco) and placed on a $100-\mathrm{cm} 2$ Petri dish for mechanical separation of the epidermis from the dermis.

For keratinocyte isolation, the epidermal pieces were placed into a $15-\mathrm{mL}$ Falcon tube containing $5 \mathrm{~mL}$ of $0.1 \%$ trypsin/0.04\% ethylenediaminetetraacetic acid (EDTA; Gibco) solution and incubated in a humidified atmosphere of 5\% $\mathrm{CO} 2$ at $37^{\circ} \mathrm{C}$ for 10 minutes. Large pieces were then removed, placed into a second $15-\mathrm{mL}$ Falcon tube containing $5 \mathrm{~mL}$ of $0.25 \%$ trypsin solution, and incubated again under the same conditions. In order to obtain a keratinocyte pellet required to start the culture, $5 \mathrm{~mL}$ of keratinocyte growth medium was added to the tube to neutralize the trypsin. The tube was centrifuged at $800 \mathrm{rpm}$ for $5 \mathrm{~min}$, the supernatant was discarded, and the keratinocyte pellet was resuspended in keratinocyte growth medium.

Keratinocytes cultures were prepared for every patient over a feeder layer of mice 3T3-J2 fibroblasts (ATCC, New York, USA), which was grown to $50 \%$ confluence incubated in a humidified atmosphere of $5 \% \mathrm{CO} 2$ at $37^{\circ} \mathrm{C}$. The keratinocyte growth medium containing the resuspended keratinocytes was then placed over the $3 \mathrm{~T} 3-\mathrm{J} 2$ culture in $75-\mathrm{cm} 2$ culture flasks.

Keratinocytes adhered to the culture flask after 24 hours. The keratinocyte growth medium was then replaced with keratinocyte growth medium supplemented with epidermal growth factor (EGF) at $1 \mathrm{\eta g} / \mathrm{mL}$ (Sigma), and the culture was incubated in a humidified atmosphere of $5 \% \mathrm{CO} 2$ at $37^{\circ} \mathrm{C}$. The keratinocyte growth medium from each culture flask was replaced every $48 \mathrm{~h}$ with $10 \mathrm{~mL}$ of keratinocyte growth medium supplemented with EGF. When cells reached $80 \%$ confluency, they were transferred to new culture flasks. Keratinocytes at passage 3 were used in the experiments.

\section{KGF supplementation}

For keratinocyte cultures in the TKB and TKC groups, the growth medium was replaced on passage 3 at $80 \%$ confluence with $15 \mathrm{~mL}$ of keratinocyte growth medium supplemented with 
KGF (Sigma) at $20 \mathrm{\eta g} / \mathrm{mL}$ (protocol adapted from Sobral et $\left.a l{ }^{27}\right)$. After $24 \mathrm{~h}$, the cells were detached from the flask and RNA extraction was performed.

\section{RNA isolation and purification}

RNA was extracted from cells using Trizol reagent (Invitrogen, Foster City, CA). Total RNA was then purified with Qiagen RNeasy Mini kit (Qiagen, Valencia, CA) and treated with DNAse. The amount and quality of the extracted RNA were assessed using a NanoVue GE Plus spectrophotometer (GE Healthcare, Cleveland, $\mathrm{OH}$ ) and a bioanalyzer (Agilent Technologies Inc., Palo Alto, CA).

\section{Quantitative polymerase chain reaction ( $q P C R)$ ar-} ray

A sample of $1 \mu \mathrm{g}$ of total RNA per array plate obtained from each group (TKB, UKB, TKC, and UKC) was used for synthesis of cDNA. Samples were treated with the buffer provided in the kit and reverse transcription reactions were performed with the $\mathrm{RT}^{2}$ First Strand kit (Qiagen). qPCR array was conducted using the $\mathrm{RT}^{2}$ Profiler PCR Arrays (Qiagen); each pool was distributed in triplicate in a $\mathrm{qPCR}$ array plate for analysis.

Eighty-four human genes related to wound healing (PAHs-121ZC) were assessed. Amplification, data acquisition, and analysis curves were performed on an ABI Prism 7500 FAST Sequence Detection System (Applied Biosystems, Foster City, CA).

The same threshold was established for all genes to ensure comparisons between curves. Three genes (ACTB - NM_001101, GAPDH - NM_002046, and HPRT1 - NM_000194) were used as normalizing genes and the mean $\mathrm{Cq}$ value was used to standardize gene expression and determine between-group differences. Values obtained from different groups were used as a reference for between-group comparisons. A 2-fold cut-off was used to identify genes differentially expressed (up-regulated or down-regulated genes).

Data analysis was performed using the $\mathrm{RT}^{2}$ Profiler PCR Array Data Analysis (http://pcrdataanalysis.sabiosciences.com/ pcr/arrayanalysis.php).

\section{Statistical analysis}

The Student's t-test was used to compare the three replicate $2^{(-\Delta c t)}$ values. Analysis of variance (ANOVA) was conducted. All statistical tests were carried out at a significance level $\alpha$ of $0.05(p<0.05)$. Also, only fold change $>2$ or $<-2$ and "OK" and "A" criteria were considered in accordance with the rules of the $\mathrm{RT}^{2}$ Profiler PCR Array Data Analysis.

\section{Results}

Eight patients participated in this study. Four women with a mean age of 42.5 years were selected as controls and 4 (3 women and 1 man) burn patients and a mean age of 36 years were also included in the study.

For all patients, gene expression was assessed by qPCR and comparisons among groups are shown in Table 1. For all comparisons, the values from the second group were always used as a reference.

TABLE 1 - Number of differentially expressed genes in relative gene expression among groups.

\begin{tabular}{llccc}
\hline Comparison & $\begin{array}{c}\text { Down- } \\
\text { regulated } \\
\text { genes }\end{array}$ & $\begin{array}{c}\text { Up- } \\
\text { regulated } \\
\text { genes }\end{array}$ & Total \\
\hline 1 & TKC versus UKC $^{1}$ & 1 & 1 & 2 \\
2 & UKB versus UKC $^{1}$ & 5 & 0 & 5 \\
3 & TKB versus UKC $^{1}$ & 5 & 0 & 5 \\
4 & TKB versus UKB $^{1}$ & 0 & 0 & 0 \\
5 & TKB versus TKC $^{1}$ & 26 & 7 & 33 \\
6 & UKB versus TKC $^{1}$ & 25 & 11 & 36 \\
\hline
\end{tabular}

$\mathrm{TKB}=\mathrm{KGF}$-treated keratinocytes from burn patients; TKC $=$ KGF-treated keratinocytes from controls; $\mathrm{UKB}=$ untreated keratinocytes from burn patients; $\mathrm{UKC}=$ untreated keratinocytes from controls.

${ }^{1}$ The second group in every comparison was always used as a reference.

Genes differentially expressed showing significant differences in expression (fold change $>2$ or $<-2$; comments " $O K$ " or "A"; $p<0.05$ ) are listed in Table 2 with respective fold change values for comparisons $1,2,3,5$ and 6 . The comparison 4 (TKB versus UKB) was not included in Table 2 because no significant difference in gene expression was found between the two groups. 
TABLE 2 - Genes differentially expressed and statistically significant in comparisons 1, 2, 3, 5, and 6.

\begin{tabular}{|c|c|c|c|c|c|c|}
\hline Genes & RefSeq & (1) TKC vs. UKC & (2) UKB vs. UKC & (3) TKB vs. UKC & (5) TKB vs. TKC & (6) UKB vs. TKC \\
\hline ACTA2 & NM_001613 & - & - & - & $-3.38 \downarrow$ & $-2.84 \downarrow$ \\
\hline COL14A1 & NM_021110 & - & - & - & $2.45 \uparrow$ & $2.91 \uparrow$ \\
\hline COL3A1 & NM_000090 & - & $-32.07 \downarrow$ & $-38.19 \downarrow$ & $-61.52 \downarrow$ & $-51.66 \downarrow$ \\
\hline COL5A1 & NM_000093 & - & - & - & $-9.12 \downarrow$ & $-7.66 \downarrow$ \\
\hline CSF3 & NM_000759 & $-69.08 \downarrow$ & $-22.18 \downarrow$ & $-26.41 \downarrow$ & - & - \\
\hline CTGF & NM_001901 & - & - & - & $-15.97 \downarrow$ & $-13.41 \downarrow$ \\
\hline CTNNB1 & NM_001904 & - & - & - & $2.26 \uparrow$ & $-47.03 \downarrow$ \\
\hline CTSK & NM_000396 & - & $-29.47 \downarrow$ & $-35.09 \downarrow$ & $-56.01 \downarrow$ & - \\
\hline FGF10 & NM_004465 & - & - & - & - & $3.00 \uparrow$ \\
\hline IFNG & NM_000619 & - & - & - & $2.50 \uparrow$ & $2.98 \uparrow$ \\
\hline IL6 & NM_000600 & - & - & - & $-5.51 \downarrow$ & $-4.62 \downarrow$ \\
\hline IL6ST & NM_002184 & - & - & - & $-7.23 \downarrow$ & $-6.07 \downarrow$ \\
\hline IL10 & NM_000572 & - & - & - & - & $2.91 \uparrow$ \\
\hline ITGA3 & NM_002204 & - & - & - & - & $2.06 \uparrow$ \\
\hline ITGA5 & NM_002205 & - & - & - & $-4.28 \downarrow$ & $-3.59 \downarrow$ \\
\hline ITGAV & NM_002210 & - & - & - & $-2.81 \downarrow$ & $-2.36 \downarrow$ \\
\hline ITGB1 & NM_002211 & - & $-23.60 \downarrow$ & $-28.11 \downarrow$ & $-31.69 \downarrow$ & $-26.61 \downarrow$ \\
\hline PDGFA & NM_002607 & - & - & - & $2.33 \uparrow$ & $2.78 \uparrow$ \\
\hline PLAU & NM_002658 & - & - & - & $2.53 \uparrow$ & $3.01 \uparrow$ \\
\hline PTEN & NM_000314 & - & - & - & $2.62 \uparrow$ & $2.50 \uparrow$ \\
\hline RAC1 & NM_006908 & - & - & - & $-7.51 \downarrow$ & $-6.31 \downarrow$ \\
\hline RHOA & NM_001664 & - & - & - & $-13.10 \downarrow$ & $-11.00 \downarrow$ \\
\hline SERPINE1 & NM_000602 & - & - & - & $-2.36 \downarrow$ & $-2.72 \downarrow$ \\
\hline TAGLN & NM_003186 & - & - & - & $-20.31 \downarrow$ & $-17.05 \downarrow$ \\
\hline TGFB1 & NM_000660 & - & - & - & $-3.76 \downarrow$ & $-3.15 \downarrow$ \\
\hline TIMP1 & NM_003254 & - & - & - & $-3.68 \downarrow$ & $-3.09 \downarrow$ \\
\hline WISP1 & NM_003882 & - & - & - & $-2.05 \downarrow$ & - \\
\hline VEGFA & NM_003376 & - & - & - & - & $3.58 \uparrow$ \\
\hline
\end{tabular}

$\uparrow=$ up-regulated gene; $\downarrow=$ down-regulated gene.

Thirty-one differently expressed genes were common to both the comparisons 5 and 6 , of which 6 were up-regulated and 25 were down-regulated genes (Table 2). There were 3 down-regulated genes [collagen type III alpha 1 (COL3A1), integrin subunit beta 1 (ITGB1), and matrix metallopeptidase 2 (MMP2)] common to comparisons $2,3,5$ and 6 . In addition, the colony stimulating 
factor (CSF3) gene was down-regulated in comparisons 1, 2 and 3, and the collagen type I alpha 2 (COL1A2) gene was up-regulated in comparison 1 , but intensely down-regulated in comparisons 5 and 6 (Table 2).

\section{Discussion}

Severe burn injury is one of the most devastating processes that a person may suffer in life. Methods for improving wound healing and tissue repair, and therapeutic interventions attenuating the acute inflammatory response and reducing the expression of cytokines after burn injury may decrease mortality, improve the quality of life of patients, and reduce health care costs ${ }^{14,15}$.

A better understanding of the therapeutic value of the growth factors may contribute to improve treatment response for burn patients ${ }^{17}$. In vitro and in vivo studies have shown the KGF activity in wound healing ${ }^{18-23}$, although Wearing and Sherratt ${ }^{15}$ found that KGF added to the epidermis decreases reepithelialization, although increases the signaling range of KGF.

This study compared the expression of wound-healingrelated genes in cultured keratinocytes from burn and healthy patients treated or not treated with KGF, as a novel approach to understand the re-epithelialization process. Papers using a similar approach were not found in the literature.

In this study, 6 different comparisons were performed among the experimental groups: (1) TKC versus UKC; (2) UKB versus UKC; (3) TKB versus UKC; (4) TKB versus UKB; (5) TKB versus TKC; and (6) UKB versus TKC. The second group of all comparisons was always used as reference for relative gene expression measurements.

Comparison 1 (TKC versus UKC) showed that the KGFtreated keratinocytes from control patients differentially expressed one down-regulated gene and one up-regulated gene compared with untreated keratinocytes from controls.

Comparison 2 (UKB versus UKC) revealed that untreated keratinocytes from burn patients differentially expressed 5 downregulated genes associated with the burn injury when compared with untreated keratinocytes from controls.

Comparison 3 (TKB versus UKC) resulted in the same 5 down-regulated genes found in comparison 2. This was the first indication that KGF supplementation was not changing gene expression in keratinocytes from patients with severe burn injury.

Comparison 4 (TKB versus UKB) was expected to show major differences in relative gene expression, resulting from the effect of KGF supplementation on keratinocytes from burn patients. However, not a single gene was differentially expressed, indicating that KGF treatment had no effect on gene expression in keratinocytes from burn injuries.

In comparisons 5 and 6 (TKB versus $\mathrm{TKC}$; and $\mathrm{UKB}$ versus TKC, respectively), the TKC group (KGF-treated keratinocytes from controls) was used as the reference group. Comparisons 5 and 6 resulted in 31 differentially expressed genes (25 down-regulated and 6 up-regulated genes) common to both groups. The genes COL3A1, ITGB1, and MMP2 were downregulated in comparisons $2,3,5$ and 6. COL3A1 encodes proalpha 1 chains of type III collagen which is found in extensible connective tissues (http://www.ncbi.nlm.nih.gov/gene/1281). To our knowledge, there is no study relating COL3A1 with burn wounds or KGF. ITGB1 encodes a beta subunit of integrins, which are membrane receptors involved in cell adhesion and recognition in a variety of processes including hemostasis, tissue repair, and immune response (http://www.ncbi.nlm.nih.gov/ gene/3688). Keely et $a l .{ }^{28}$ identified ITGB1 as a target gene of hypoxia-inducible factor (HIF), a global regulator of oxygen homeostasis. The authors suggested that ITGB1 contributes to the wound-healing process in both the extracellular matrix (ECM) and epithelium, and found that hypoxia induces fibroblast ITGB1 expression. It is known that burn wounds are associated with tissue hypoxia, but our findings showed that the ITGB1 gene was down-regulated in keratinocytes from burn patients, in contrast to the hypothesis of Keely et al. ${ }^{28}$. MMP2 encodes a type IV collagenase, capable of cleaving components of the extracellular matrix and molecules involved in signal transduction (http://www. ncbi.nlm.nih.gov/gene/4313). Kubo et al. ${ }^{29}$ showed that MMP2 expression increased from day 3 to day 14 postburn. Zhao et al. ${ }^{30}$ suggested that angiogenesis depends on MMP2, which is secreted by activated endothelial cells, and showed that MMP2 expression increases between days 1 and 3 postburn, decreasing after day 7. In this study, the MMP2 gene was down-regulated at day 3 postburn, in contrast to Kubo et al. ${ }^{29}$ and Zhao et al. ${ }^{30}$ findings. Further studies are necessary to investigate the expression of MMP2in burn wounds. CSF3 encodes a cytokine that controls the production, differentiation, and function of granulocytes (http:// www.ncbi.nlm.nih.gov/gene/1440). In this study, CSF3 was down-regulated in the comparisons 1,2 and 3. To date, no studies were found relating CSF3 with burn wounds.

Therefore, KGF supplementation did not change the relative gene expression in comparisons 2 and 3 and comparisons 
5 and 6, showing no significant difference associated with KGF treatment in cultured primary keratinocytes from patients with severe burn injuries.

\section{Conclusions}

There was no differential expression of wound-healingrelated genes in cultured primary keratinocytes from burn patients treated with keratinocyte growth factor (KGF). Clinically, the action of KGF may be controversial for the re-epithelialization in acute severe burns in the skin close by the eschar. It takes more experimental and clinical studies to evaluate the effects of KFG at re-epithelialization in burns injury.

\section{References}

1. Penn JW, Grobbelaar AO, Rolfe KJ. The role of the TGF- $\beta$ family in wound healing, burns and scarring: a review. Int J Burns Trauma. 2012;2:18-28. PMID: 3415964.

2. American Burn Association (ABA). National Burn Repository: 2014 Report. Available from http://ameriburn. org/2014NBRAnnualReport.pdf; 2014.

3. Iurk LK, Oliveira AF, Gragnani A, Ferreira LM. Evidence-based medicine in burns treatment. Rev Bras Queimaduras. 2010;9:95-9. doi: 10.1097/BCR.0b013e318191fd95.

4. Wolf SE, Tompkins RG, Herdon DN. On the horizon: research priorities in burns for the next decade. Surg Clin North Am. 2014;94:917-30. doi: 10.1016/j.suc.2014.05.012.

5. Hettiaratchy S, Dziewulski P. ABC of burns: pathophysiology and types of burns. BMJ. 2004;328:1427-9. doi: 10.1136/ bmj.328.7453.1427.

6. Gragnani A, Dell'Aquila AM, Doi AM, Müller BR, Lacerda LA, Machado AMO, et al. Microbiological surveillance of burn unit of EPM/UNIFESP in São Paulo, Brazil. Rev Bras Cir Plast. 2014;29:114-9. doi:10.5935/2177-1235.2014RBCP0019.

7. Noronha SA, Noronha SM, Lanziani LE, Ferreira LM, Gragnani A. Innate and adaptive immunity gene expression of human keratinocytes cultured of severe burn injury. Acta Cir Bras. 2014;29:60-7. doi: 10.1590/s0102-86502014001700012.

8. Shupp JW, Nasabzadeh TJ, Rosenthal DS, Jordan MH, Fidler P, Jeng JC. A review of the local pathophysiologic bases of burn wound progression. J Burn Care Res. 2010;31:849-73. PMID: 21105319.

9. Gragnani A, Cezillo MV, da Silva ID, de Noronha SM, CorreaNoronha SA, Ferreira LM. Gene expression profile of cytokines and receptors of inflammation from cultured keratinocytes of burned patients. Burns. 2014;4:947-56. doi: 10.1016/j.burns.2013.11.022.

10. Gibran NS, Heimbach DM. Current status of burn wound pathophysiology. Clin Plast Surg. 2000;27:11-22. PMID: 10665353.

11. Arturson G. Pathophysiology of the burn wound. Ann Chir Gynaecol. 1980;69:178-90. PMID: 6162412.

12. Yang Q, Orman MA, Berthiaume F, Ierapetritou MG, Androulakis IP. Dynamics of short-term gene expression profiling in liver following thermal injury. J Surg Res. 2012;176:549-58. doi: 10.1016/j.jss.2011.09.052.

13. Herndon DN, Tompkins RG. Support of the metabolic response to burn injury. Lancet. 2004;363:1895-902. doi: 10.1016/S01406736(04)16360-5.
14. Baxter RM, Dai T, Kimball J, Wang E, Hamblin MR, Wiesmann WP, McCarthy SJ, Baker SM. Chitosan dressing promotes healing in third degree burns in mice: Gene expression analysis shows biphasic effects for rapid tissue regeneration and decreased fibrotic signaling. J Biomed Mat Res A. 2013;101:340-8. doi: 10.1002/jbm.a.34328.

15. Wearing HJ, Sherratt JA. Keratinocyte growth factor signalling: a mathematical model of dermal-epidermal interaction in epidermal wound healing. Math Biosci. 2000;165:41-62. doi: 10.1016/S00255564(00)00008-0.

16. Greaves NS, Ashcroft KJ, Baguneid M, Bayat A. Current understanding of molecular and cellular mechanisms in fibroplasia and angiogenesis during acute wound healing. J Dermatol Sci. 2013;72:206-17. doi: 10.1016/j.jdermsci.2013.07.008. doi: 10.1016/j.jdermsci.2013.07.008.

17. Ching YH, Sutton TL, Pierpont YN, Robson MC, Payne WG. The use of growth factors and other humoral agents to accelerate and enhance burn wound healing. Eplasty. 2011;11:e41. PMID: 22084646.

18. auf demKeller U, Krampert M, Kümin A, Braun S, Werner S. Keratinocyte growth factor: effects on keratinocytes and mechanisms of action. Eur J Cell Biol. 2004;83:607-12. doi: 10.1078/0171-933500389.

19. Werner S, Smola H, Liao X, Longaker MT, Krieg T, Hofschneider PH, Williams LT. The function of KGF in morphogenesis of epithelium and reepithelialization of wounds. Science. 1994;266:819-22. PMID: 7973639.

20. Marti G, Ferguson M, Wang J, Byrnes C, Dieb R, Qaiser R, Bonde P, Duncan MD, Harmon JW. Electroporative transfection with KGF-1 DNA improves wound healing in a diabetic mouse model. Gene. 2004;11:1780-5. PMID: 15470477.

21. Staiano-Coico L, Krueger JG, Rubin JS, D'limi S, Vallat VP, Valentino L, Fahey T 3rd, Hawes A, Kingston G, Madden MR, Mathwich M, Gottlieb A, Aaronson SA. Human keratinocyte growth factor effects in a porcine model of epidermal wound healing. J Exp Med. 1993;178:865-78. PMID: 8350059.

22. Pierce GF, Yanagihara D, Klopchin K, Danilenko DM, Hsu E, Kenney WC, Morris CF. Stimulation of all epithelial elements during skin regeneration by keratinocyte growth factor. J Exp Med. 1994;179:831-40. PMID: 7509362.

23. Kopp J, Wang GY, Kulmburg P, Schultze-Mosgau S, Huan JN, Ying K, Seyhan H, Jeschke MD. Accelerated wound healing by in vivo application of keratinocytes overexpressing KGF. Mol Ther. 2004;10:86-96. PMID: 15233945.

24. Green H, Kehinde O, Thomas J. Growth of cultured human epidermal cells into multiple epithelia suitable for grafting. Proc Natl Acad USA. 1979;76:5665-8. PMID: 293669.

25. Green H. Cultured cells for the treatment of disease. Sci Am. 1991;265:96-102. PMID: 1785043.

26. Hirsch T, von Peter S, Dubin G, Mittler D, Jacobsen F, Lehnhardt M, Eriksson E, Steinau HU, Steinstraesser L. Adenoviral gene delivery to primary human cutaneous cells and burn wounds. Mol Med. 2006;12:199-207. doi: 10.2119/2006-00031.Hirsch

27. Sobral CS, Gragnani A, Morgan J, Ferreira LM. Inhibition of proliferation of Pseudomonas aeruginosa by KGF in an experimental burn model using human cultured keratinocytes. Burns. 2007;33:613-20. PMID: 17418954.

28. Keely S, Glover LE, MacManus CF, Campbell EL, Scully MM, Furuta GT, Colgan SP. Selective induction of integrin beta1 by hypoxia-inducible factor: implications for wound healing. FASEB J. 2009;23:1338-46. PMID: 19103643.

29. Kubo H, Hayashi T, Ago K, Ago M, Kanekura T, Ogata M. Temporal expression of wound healing-related genes in skin burn injury. Leg Med (Tokyo). 2014;16:8-13. PMID: 24269074. 
Chomiski V et al.

30. Zhao J, Chen L, Shu B, Tang J, Zhang L, Xie J, Qi S, Xu Y. Granulocyte/macrophage colony-stimulating factor influences angiogenesis by regulating the coordinated expression of VEGF and the Ang/Tie system. PloS One. 2014;9:e92691. PMID: 24658178.

\section{Correspondence:}

Alfredo Gragnani

Rua Napoleão de Barros, $715 / 4^{\circ}$ andar

04024-002 São Paulo - SP Brasil

Tel.: (55 11)5576-4118 / 5579-4933

Fax: (55 11)5571-6579

agragnanif@gmail.com

Received: Apr 28, 2016

Review: Jun 23, 2016

Accepted: July 21, 2016

Conflict of interest: none

Financial source: FAPESP (number 2013/10905-0)

${ }^{1}$ Research performed at Division of Plastic Surgery, Department of Surgery, Universidade Federal de São Paulo (UNIFESP), Brazil. 